\title{
Crow Search Algorithm (CSA)
}

\author{
Suvarna Shirke, R. Udayakumar
}

\begin{abstract}
Researchers' are taking keen interest in Optimization algorithms due to their heuristic and meta-heuristic nature. Heuristic algorithms find the arrangement by utilizing the experimentation strategy. Then again, meta-heuristic algorithms discover the response at a more elevated tier. Several nature-based metaheuristic algorithms are easily accessible. Askarzadeh has introduced the Crow search algorithm and stated that it is meta-heuristic optimization algorithm. The astute conduct of the crow moves CSA. Crows are keen on putting away the abundance nourishment at concealing spots and recuperating it at whatever point it is needed. CSA's previous outcomes show that it can unravel different complex building related optimization issues. There are six compelled building plan issues, and CSA is utilized to upgrade these issues. This paper focuses on a far-reaching investigation of CSA in the diverse application is given with the examination just as the exhibitions of the CSA in the different structure is talked about.
\end{abstract}

Keywords: Metaheuristic Algorithms, Crow Search Algorithm, Earthworm Algorithm

\section{INTRODUCTION}

There are various heuristic and metaheuristic optimization algorithms open. The heuristic count wears down experimentation. It is also observed that metaheuristic estimation works at a progressively exceptional stage. The metaheuristic algorithms which are Nature- are isolated into four sorts: advancement based systems, material science-based methodology, swarm-based methodologies, and human-based strategies. The cases of the metaheuristic optimization algorithms are [6] [10]:

1. Firefly Algorithm

2. Ant Colony Optimization (ACO)

3. Simulated Annealing (SA)

4. Artificial Bee Colony

5. Cuckoo Search Algorithm

6. Cat Swarm Optimization

7. Tabu Search

8. Genetic Algorithm (GA)

9. Bat Algorithm

10. Wolf Search Algorithm

11. Gravitational Search Algorithm

12. Crow Search Algorithm (CSA)

13. EarthWorm Algorithm(EWA)

14. Particle Swarm Optimization
15. Krill Herd

The metaheuristic algorithm types are depicted in Figures 1 and 2 .

Affirmations of Crows being one of the most insightful feathered animals are bountiful. The best talent they have is they can recall the faces and alert each other. Through their brain to body extent is tall, due to this feature they can fly high. From a long time crows are considered as cheat. Crows watch the fortresses of various fowls as soon as the owner left they obtain the food. Crows get the knowledge from their earlier experiences, so they do not take any risk to change their nook to refuse to be a future terrible setback.

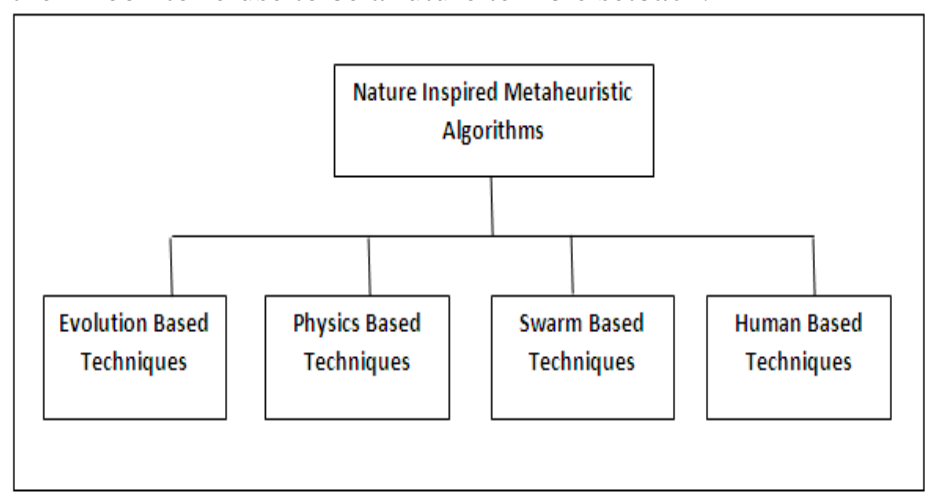

Fig. 1. Example of Nature-Inspired Metaheuristic Algorithms

Crow Search Algorithm is introduced late as a meta-heuristic analyzer. CSA concludes from the vigilant lead of crows. CSA uses the core thinking of "How crow stock up their plenitude sustenance in their shelters and recuperate a comparative when crucial?" CSA is associated with improving the six constrained structure plan, and it has restrictions and decision factors. The benchmarks of CSA are that Crows live in cluster and they hold the situation of their hiding regions. Crows also check each other's lousy behavior, and they protect their food from getting stolen.

Crow Search Algorithm accomplishes the perfect strategy from the hunt space.

Establish the elements of group gauge, length of the fight, care probability, plus the majority extraordinary accentuations.

$>$ Establish the crow position and its memory

$>$ Goal work evaluated.

$>$ Creation of the new position is done.

$>$ Now examine that the new position is attainable or not.

$>$ Perform the new positions assessment.

$>$ Lastly memory is refreshed.

Revised Manuscript Received on October 25, 2019

Suvarna Shirke, Research Scholar, Department of Computer Science and Engineering, Bharath Institute of Higher Education and Research, Chennai, India. Assistant Professor at Atharva College of Engineering.

Dr.R.Udayakumar, Professor and Supervisor, Department of Information Technology, Bharath Institute of Higher Education and Research, Chennai, India 


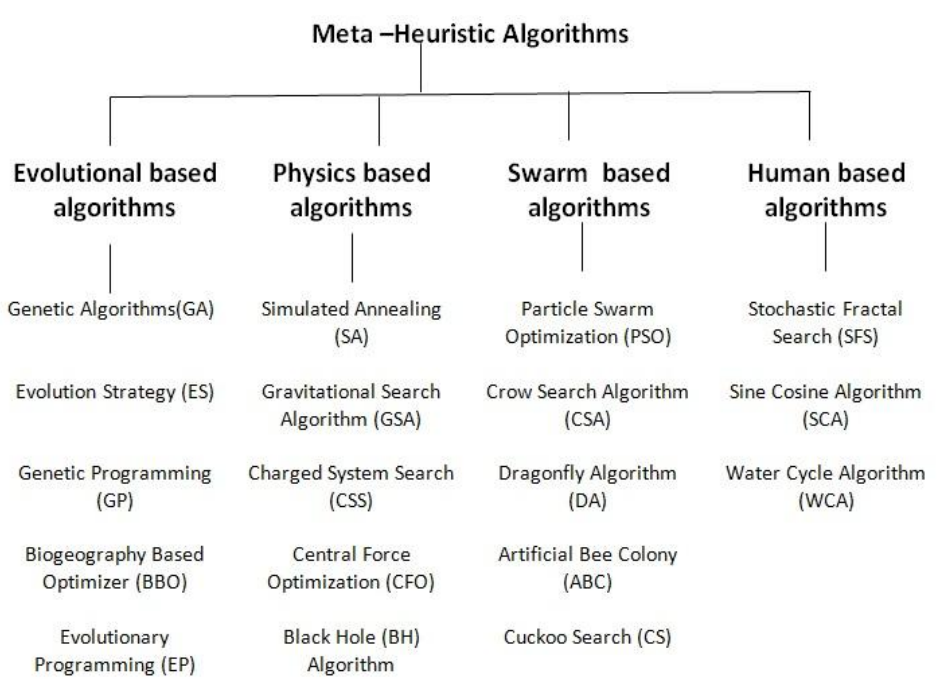

Fig.2. Taxonomy of metaheuristic algorithms.

By driving the above advances, the calculation hopes to explore through the inquiry space and find a perfect game plan. It has an incredible broadness of usage yet a couple of issues of much of the time finding the close by precise and working with high dimensionality enlightening list needs some tweaking of the going with calculation, the work for which is examined underneath:

\section{LITERATURE SURVEY}

Network performance improvement is an essential issue in networking. Almost and Ahmed[13] have developed the conductor size selection process for the radial distribution network for increasing the network performance. If the conductor size is selected, then it results in a small amount of power loss and enhances the bus voltage. It helps to reduce the system's yearly operating cost. Authors have developed an objective function, which is a summation of conductor assets cost as well as the cost of conductor energy loss. The constraints used are restricted bus voltage and conductor's current capability. They have used independent variables such as conductor's type and size and minimize the objective function. The developed method evaluated on 2 network topologies. There are 16 bus systems in first topology and a large scale 85 bus system in the second bus topology.

Shady et al.[ 12] Have proposed third-order damped filter where they have used unequal capacitors, and also that derive the relationship between the circuit parameters. They have formulated the optimal design problem of the filters for minimizing the filter cost by considering the investment and the operating expenditure. The constraints they have considered are the total and individual harmonic distortion indices, voltage amplification ratios used for measuring the filter resonance damping capability, and power factor.CSA used for finding the result for formulated design problem. Here, authors have made the comparison of designs with third-order access filters and third-order C type filter. Designed filter assures no electrical resonance hazards the performance parameters like load and filter are maintained in the given limit. $\mathrm{C}$ type filter has given the better efficiency for high power factor. It also enhanced loss in transmission as compare to other 2 filters. New designed filter has the identical current and voltage harmonic improvement levels.
In the worst case scenario $\mathrm{C}$ type filter and third order high pass filter

The solution for the cost minimization problem also reveals that under the worst case of conditions, the $\mathrm{C}$ type filter has worst resonance damping capabilities if the capacitors are same. The third order high pass filter has best resonance damping capabilities if the capacitors are same. The authors also compared the crow search algorithm with a genetic algorithm and the Particle Swarm Optimisation technique. The system is valid and has a fast convergence capability.

Modified Crow Search Algorithm (MCSA) introduced by Deepak Get al. [11]. This algorithm used for feature extraction usability and prediction. The software usability models which are hierarchical consider usability as an essential aspect. Extension to CSA is a MCSA. The Modified Crow Search Algorithm (MCSA) algorithm focuses on to extract the usability features from the hierarchical model through best potential solution. After obtaining the features, MCSA generates a particular number of attributes, and these generated attributes applied to the Software Development Life Cycle (SDLC) models to get the finest among them. The MCSA compared with the original Binary Bat Algorithm (BBA), CSA, and whale optimization algorithm (MWOA). The performance of MCSA is enhanced and generated additional feature selection.

Ali and Farhad [10] have proposed a crow look calculation for content report arrangement. Here, they have characterized the archives from much information. The time expected to look through the necessary information, so to lessen the time, the characterization of related materials is done in a particular class. Feature selection is performed wsing CSA and KNN is used for grouping.

Also, for tallying words and figuring the recurrence of words TF method is utilized. The dataset on which the investigation is done is Cade 12, Webkb, and Reuter-21578. The datasets used for assessment contains the accompanying information. It comprises of three datasets named Reuters-21578, Webkb, and Cade 12. The Reuters-21578 dataset has 21578 messages in 135 distinct classes. The Webkb dataset has Set of different archives on the web, various materials gathered from multiple Computer Science Departments of colleges, and 4199 content records. The VC\#.NET 2015 framework I used for execution. The correctness of the structure is enhanced.

Swati et al. [9] have implemented CSA calculation for an optimization issue. They have performed reproductions on various numerical benchmark works on few practical building structure issues. Implementation of the proposed system is executed utilizing Matlab, and the consequences of the context are contrasted and a number of current metaheuristic methodologies. Furthermore, the impact of the control parameter's progress on the presentation of CSA is the center of the paper. The CSA is discovered better for correct building issues over some conventional heuristic calculation on account of depend on the type of the problem, the similar examination capacity, and simple execution on MATLAB. 
CSAK calculations are introduced by Lakshmi et al. [8]. In this CSA is joined with the $\mathrm{K}$-implies grouping calculation to obtain the worldwide ideal arrangement. On six datasets, the CSAK evaluation is done. These databases are Iris, Glass, Wine, Contraceptive Method Choice, Haberman's Survival, and Wisconsin Breast Cancer. The wine dataset has the compound assessment of wines created in a comparable region, be that as it may, it is obtained from three exciting cultivar. In the wine 13 qualitites are found in all the 3 types of wines. The Wisconsin Breast Cancer dataset contains wedded ladies' tests who are either not pregnant or do not know at the hour of the meeting. It is utilized to foresee the immediate preventative strategy decision like extended haul techniques, momentary strategies, and no utilization. It uses the ladies' statistics and financial attributes. For every determination, there are nine amounts. Iris dataset has 150 instances of iris bloom with three one of a kind animal categories. The species join Versicolour,Setosa, and Virginica. Per specie 50 observations are there. The characteristics in each species are sepal length, sepal width, petal length, and petal width.

Prophylactic Method Choice (CMC) database utilized The CMC dataset is used to discover the sort of bosom malignant growth. There are two kinds of bosom tumors, and for each class, nine amounts are found. Haberman's Survival dataset has the information of the patients who made due after bosom malignancy medical procedure. The source of information used is the University of Chicago's Billings Hospital. It has the investigation data in the range of 1958 and 1970. Glass dataset comprises of the sorts of glass persuaded by criminological assessment. In the area of the bad behavior, the display left can be used as confirmation, in case it is precisely recognized. There are ten amounts found in all of the six sorts of glass.

The whole Crow Search Algorithm (HWAC) is introduced by Ze-Xue et al. [7]. This technique has used the Central optimization process. The up-gradation of the arrangement is to dine based on CPSO estimation. The HWAC has two administrators: Individual Hybrid administrator and improved variety administrator. HWAC utilizes Euclidean separation as a wellness capacity to discover similitudes between two bunches (examples) and to discover the separation between them. Worldwide Evolution is a blend of CSA and WOA positions to get another area. The roulette wheel approach is used for structure enhancement. A structure of Hybrid individual is utilized at open cycles for selecting a particular framework and replace it with two swarm-based structures.

A variety grows, the administrator is like the hybrid administrator, as in the DE calculation. Examination aftereffects of the HWCA calculation on the UCI database show huge increment incorrectness of the framework, expanding to $18 \%$ when contrasted with WOA and up with $8 \%$ if there should arise an occurrence of the CSA calculation.

Rough Crow Search Algorithm (RCSA) is introduced by Aboul [5]. It is a half and a half-intelligent algorithm, it means it is half CSA and half rough searching plan, and due to this, it is taking care of the number of optimization problems. The RCSA algorithm combines excellent features from the two algorithms and tries to find out the region of the global solution. The high dimensionality data has roughness and inaccuracy issues, and these issues are solved by fusing the RSS. The computational cost needed for RCSA is low as it approximates the upper-lower approximations and speeds up the execution of the task. The outcome of the RCSA is contrast. Different benchmarking measures and calculations and the connections show that the RCSA defeats interchange calculations all benchmark issues comparatively as a result bore subordinate upon the effects from ensuring quantifiable measures and Wilcoxon marked positions to examination.

The combined algorithms give rise to the low precision problem as well as lesser accuracy in optimization. So a new algorithm Improvement in CSA (ICSA) [4] is introduced. The dimensional optimization issues are handled very efficiently by ICSA. The factors consisder for misuse and testing limit of CSA are versatile change administrator, and Levy flight new experience factor. The supervisor and the Levy flight distribution set uo the in position updation of the crow. The determined examination of search space is progressed by the Levy flight circulation similarly as it keeps problematic blend by evading from neighborhood perfect at any stage. They attempted the execution of ICSA on high-dimensional nonlinear versatile benchmark test abilities to find that the proposed calculation is outstandingly engaged and less sensitive to work estimations. ICSA furthermore performed with predominance over other established reformation specialists. A high dimensional nonlinear versatile benchmark is used for ICSA evaluation.

A Sine Cosine Crow Search Algorithm (SCCSA) is developed by Pasandideh and Soheyl [3] for handling global optimization.

It is a half and half calculation of CSA. SCCSA is projected to handle the issues related to global optimization. The administrator of the two estimates is merged, and the SCCSA makes a tradeoff among examination and misuse frameworks. The performance of the SCCSA is surveyed against seven benchmark limits. The intermixing plots of the calculations showed that the SCCSA converges to the perfect course of action without getting in neighborhood optima. Figure 3 shows the two dimensional (2D) benchmark limits used for the test.
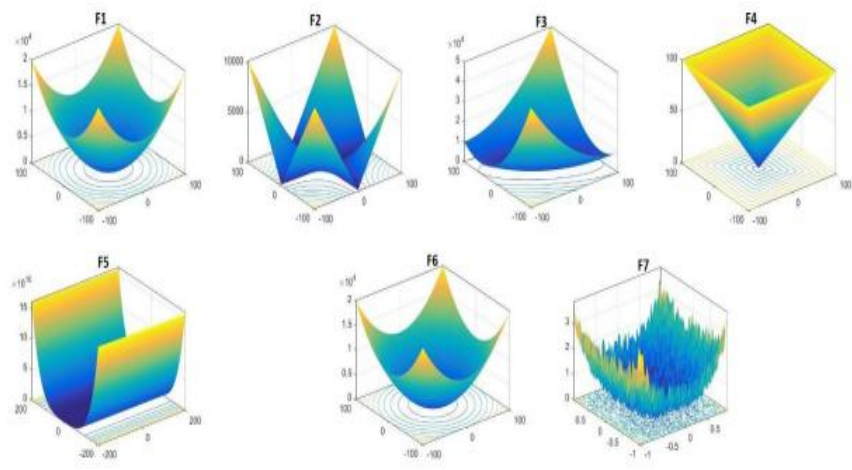

Fig. 3. Benchmark functions Two Dimensional 2D view[10] 
Planning issues are solved by Adhi A. [2] by using CSA. Choosing an asset is a crucial thing in planning the business. CSA handles this NP multifaceted nature issue. CSA beats the customary planning algorithms. Inventors have recommended that the Quantum strategy could be a choice to flee from neighborhood ideal. Estimation consequences are handled by this method. The crows' situation can be recovered if the result of the few phases is the same. CSA is a fantastic technique for giving an ideal arrangement. Time estimation is the most significant benefit of this method.

To beat the low union and the issues of the neighborhood optima, Sayed G. et al. [1] think of a half, and half calculation is known as the Chaotic Crow Search Algorithm (CCSA). The confused factors are utilized to supplant the irregular elements of the CSA. CCSA makes include determination, and the disordered succession is implanted in their pursuit cycles. The half and half calculation are tested for 20 benchmarking databases, recorded six execution criteria, and contrasted the proposed estimation and the additional meta-heuristic algorithms; they discovered that the proposed calculation outflanks others as far as best and worst wellness esteem. One of their discoveries was equally that the crossbreed CCSA close by sine map regarding arrangement execution, Stability quality, and intermingling spee.

TABLE I. Summary of the CSA in various applications.

\begin{tabular}{|c|c|c|}
\hline $\begin{array}{l}\text { Pape } \\
\mathbf{r}\end{array}$ & Method & Application \\
\hline [13] & CSA & $\begin{array}{l}\text { The proposed system is used to } \\
\text { increase the performance of the } \\
\text { network. }\end{array}$ \\
\hline [12] & CSA & $\begin{array}{l}\text { A third-order partial filter is } \\
\text { designed using CSA. }\end{array}$ \\
\hline [11] & $\begin{array}{l}\text { CCSA } \\
\text { (Chaotic } \\
\text { Crow Search } \\
\text { Algorithm) }\end{array}$ & $\begin{array}{l}\text { CCSA is used for Low } \\
\text { convergence and local optima } \\
\text { problem. The twenty benchmark } \\
\text { database and another } \\
\text { metaheuristic algorithm are used } \\
\text { to test the performance. }\end{array}$ \\
\hline$[10]$ & $\begin{array}{l}\text { Crow Search } \\
\text { Algorithm } \\
\text { (CSA) }\end{array}$ & $\begin{array}{l}\text { CSA used for scheduling problems } \\
\text { in the industry. }\end{array}$ \\
\hline [9] & $\begin{array}{l}\text { SCCSA (Sine } \\
\text { Cosine Crow } \\
\text { Search } \\
\text { Algorithm) }\end{array}$ & $\begin{array}{l}\text { SCCSA solves Global } \\
\text { optimization problems. The } \\
\text { performance of SCCSA evaluated } \\
\text { on seven benchmark functions. }\end{array}$ \\
\hline [8] & $\begin{array}{l}\text { ICSA } \\
\text { (Improved } \\
\text { Crow Search } \\
\text { Algorithm ) }\end{array}$ & $\begin{array}{l}\text { ICSA solves High dimensional } \\
\text { issues. The performance of the } \\
\text { algorithm is evaluated on } \\
\text { high-dimensional nonlinear } \\
\text { scalable benchmark test functions }\end{array}$ \\
\hline [7] & $\begin{array}{l}\text { RCSA } \\
\text { (Rough Crow } \\
\text { Search } \\
\text { Algorithm) }\end{array}$ & $\begin{array}{l}\text { RCSA is used for Multiple } \\
\text { optimization problems. The } \\
\text { performance is evaluated on } 25 \\
\text { CEC' } 2005 \text { benchmark functions. }\end{array}$ \\
\hline [5] & $\begin{array}{l}\text { WCSA } \\
\text { (Whole Crow } \\
\text { Search } \\
\text { Algorithm) }\end{array}$ & $\begin{array}{l}\text { WCSA is used for Data } \\
\text { Clustering. Evaluation is done o } \\
\text { UCI dataset }\end{array}$ \\
\hline [4] & Crow Search & CSA does Data Clustering using K \\
\hline
\end{tabular}

\begin{tabular}{|l|l|l|}
\hline & $\begin{array}{l}\text { Algorithm } \\
\text { with K-means } \\
\text { Clustering }\end{array}$ & $\begin{array}{l}\text { means clustering. Six datasets } \\
\text { used for performance evaluation. } \\
\text { The datasets are Glass, Wine, } \\
\text { Contraceptive Method Choice, } \\
\text { Wisconsin Breast Cancer, Iris, and } \\
\text { Haberman's Survival database. }\end{array}$ \\
\hline$[3]$ & $\begin{array}{l}\text { Crow Search } \\
\text { Algorithm } \\
\text { (CSA) }\end{array}$ & $\begin{array}{l}\text { CSA solves an Optimization } \\
\text { problem }\end{array}$ \\
$\begin{array}{l}\text { Crow Search } \\
\text { Algorithm }\end{array}$ & $\begin{array}{l}\text { Here CSA does Document } \\
\text { Classification. Performance } \\
\text { evaluated on Webkb dataset, Cade } \\
\text { 12 dataset, and Reuter-21578 } \\
\text { dataset }\end{array}$ \\
\hline$[1]$ & $\begin{array}{l}\text { Modified } \\
\text { Crow Search } \\
\text { Algorithm } \\
\text { (MCSA) }\end{array}$ & $\begin{array}{l}\text { It used for Feature Extraction and } \\
\text { prediction. Here, MCSA } \\
\text { compared with original CSA, } \\
\text { whale optimization algorithm } \\
\text { (MWOA), and standard binary bat } \\
\text { algorithm (BBA) }\end{array}$ \\
\hline
\end{tabular}

\section{CONCLUSION}

The crow search calculation being a meta-heuristic calculation, is an exceptionally proficient calculation in finding an ideal arrangement through a pursuit space. The estimate has a few issues with versatility with multi-modular information and now and then has a low assembly rate. The calculation here and their requirements to locate the neighborhood optima instead of the global optima.

Accordingly, tweaking the calculation and alterations performed by the above specialists help the exhibition of the CSA exponentially. As expressed above, we can see various methodologies taken by the specialists to use the productivity of this metaheuristic approach while maintaining a strategic distance from the entanglements of the equivalent.

Presentation of the knowledge factor, versatile modification administrator, and Levy flight updation system while refreshing the crow position was one of only a handful, not many methodologies taken by the analysts that demonstrated to be less touchy to practical measurements. Specialists took another proposition by the presentation of turbulent factors additionally improved the exhibition as far as strength and assembly rate. Not many different specialists contrived half breed algorithms by consolidating CSA with algorithms like Since Cosine calculation, K-Means and, RSS, to manage the issues that CSA sees.

The uses of CSA in distributed computing, identification of Parkinson at $100 \%$ exactness at a beginning time, removing highlights for programming ease of use, shows the full extent of the calculation. It would be a sensible finding that CSA has a large extension, and clubbing it with the correct systems can achieve remarkable outcomes. 


\section{REFERENCES}

[1] Sayed, Aboul, and Ahmad, "Feature selection via a novel chaotic crow search algorithm," Neural computing and applications, pp. 171-188, January 2019.

[2] Ali Allahverdipoor and Farhad Soleimanian Gharehchopogh, "An Improved K-Nearest Neighbor with Crow Search Algorithm for Feature Selection in Text Documents Classification," Journal of Advances in Computer Research, Vol.9, No.2, pp. 37-48, May 2018.

[3] Swati Rajput, Monika Parashar, Hari Mohan Dubey, Manjaree Pandit, "Optimization of benchmark functions and practical problems using Crow Search Algorithm," 2016 Fifth International Conference on Eco-friendly Computing and Communication Systems (ICECCS), IEEE, Bhopal, India, December 2016.

[4] Mohit Jain, Asha Rani, and Vijander Singh, "An improved Crow Search Algorithm for high-dimensional problems," Journal of Intelligent \& Fuzzy Systems, pp. 3597-3614,2017.

[5] Aboul Ella Hassanien, Rizk M. Rizk-Allah, and Mohamed Elhoseny, "A hybrid crow search algorithm based on a rough searching scheme for solving engineering optimization problems," Journal of Ambient Intelligence and Humanized Computing, pp. 1-25, June 2018.

[6] Arockia Panimalar.S, "Nature-Inspired Metaheuristic Algorithms," International Research Journal of Engineering and Technology (IRJET), Vol 4, Issue 10, October 2017

[7] Ze-Xue Wu, Ko-Wei Huang, Abba Suganda Girsang, "A Whole Crow Search Algorithm for Solving Data Clustering," In 2018 Conference on Technologies and Applications of Artificial Intelligence (TAAI), pp. 152-155, IEEE, November 2018.

[8] K. Lakshmi, N. Karthikeyani Visalakshi, and S. Shanthi, "Data clustering using K-Means based on Crow Search Algorithm," Sadhana, pp.190, 2018

[9] Pasandideh Seyed Hamid Reza, and Soheyl Khalilpourazari, "Sine Cosine Crow Search Algorithm: A powerful hybrid metaheuristic for global optimization," arXiv preprint arXi,2018.

[10] Adhi Antono, Budi Santosa, and Nurhadi Siswanto, "A meta-heuristic method for solving scheduling problem: crow search algorithm," IOP Conference Series: Materials Science and Engineering, Vol. 337. No. 1. IOP Publishing, 2018.

[11] Deepak Gupta, Joel J. P. C. Rodrigues, Shirsh Sundaram, Ashish Khanna, Valery Korotaev, Victor Hugo C. de Albuquerque, "Usability feature extraction using modified crow search algorithm: a novel approach," Neural Computing and Applications, pp. 1-11, August 2018.

[12] Aleem Shady, Ahmed, Murat, "Optimal resonance-free third-order high-pass filters based on minimization of the total cost of the filters using Crow Search Algorithm," Electric Power Systems Research 151 (2017): 381-394.

[13] Almoataz and Ahmed Fathy, "A novel approach based on crow search algorithm for optimal selection of conductor size in radial distribution networks," Engineering Science and Technology, an International Journal 20.2 (2017): 391-402.

\section{AUTHORS PROFILE}

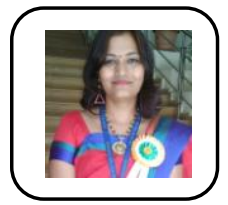

Suvarna Shirke-Pansambal is the Research Scholar at Bharath Institute of Higher Education and Research, Department of Computer Science \& Engineering, Chennai. She is working as an Assistant Professor in Atharva College of Engineering, Mumbai.

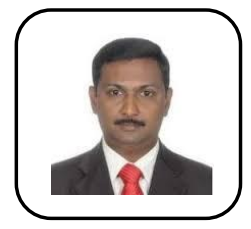

Dr.R.Udayakumar is Professor at Department of Information Technology, Bharath Institute of Higher Education and Research, Chennai, India. He has published more than 600 papers and he is Supervisor for Research Scholars at BIHER. 